\title{
Comparison of Three Algorithms to Estimate Tree Stem Diameter from Terrestrial Laser Scanner Data
}

\author{
Joris Ravaglia ${ }^{1, *, \dagger}$, Richard A. Fournier ${ }^{2}$, Alexandra Bac ${ }^{1}$, Cédric Véga ${ }^{3}$, Jean-François Côté ${ }^{4}$ (D), \\ Alexandre Piboule ${ }^{5}$ (D) and Ulysse Rémillard ${ }^{2}$ \\ 1 G-Mod, Laboratoire d'Informatique et des Systèmes UMR CNRS 7020, Aix-Marseille Université, \\ 13288 Marseille CEDEX 09, France \\ 2 Department of Applied Geomatics, Centre d'Applications et de Recherches en Télédétection, \\ Université de Sherbrooke, Sherbrooke, QC J1K 2R1, Canada \\ 3 Institut National de l'information Géographique et Forestière, Laboratoire d'Inventaire Forestier, \\ 54000 Nancy, France \\ 4 Canadian Wood Fibre Centre, Canadian Forest Service, Natural Resources Canada, \\ Québec, QC G1V 4C7, Canada \\ 5 Pôle Recherche Développement Innovation, Office National des Forêts, 54500 Villiers-lès-Nancy, France \\ * Correspondence: joris.ravaglia@univ-amu.fr \\ + Current address: LIS UMR CNRS 7020, Aix Marseille Université, Campus de Luminy-163 Avenue de \\ Luminy, Case 901, BP 5, 13288 Marseille CEDEX 9, France.
}

Received: 1 April 2019; Accepted: 15 July 2019; Published: 18 July 2019

\begin{abstract}
Terrestrial laser scanners provide accurate and detailed point clouds of forest plots, which can be used as an alternative to destructive measurements during forest inventories. Various specialized algorithms have been developed to provide automatic and objective estimates of forest attributes from point clouds. The STEP (Snakes for Tuboid Extraction from Point cloud) algorithm was developed to estimate both stem diameter at breast height and stem diameters along the bole length. Here, we evaluate the accuracy of this algorithm and compare its performance with two other state-of-the-art algorithms that were designed for the same purpose (i.e., the CompuTree and SimpleTree algorithms). We tested each algorithm against point clouds that incorporated various degrees of noise and occlusion. We applied these algorithms to three contrasting test sites: (1) simulated scenes of coniferous stands in Newfoundland (Canada), (2) test sites of deciduous stands in Phalsbourg (France), and (3) coniferous plantations in Quebec, Canada. In most cases, the STEP algorithm predicted diameter at breast height with higher R2 and lower RMSE than the other two algorithms. The STEP algorithm also achieved greater accuracy when estimating stem diameter in occluded and noisy point clouds, with mean errors in the range of $1.1 \mathrm{~cm}$ to $2.28 \mathrm{~cm}$. The CompuTree and SimpleTree algorithms respectively produced errors in the range of $2.62 \mathrm{~cm}$ to $6.1 \mathrm{~cm}$ and $1.03 \mathrm{~cm}$ to $3.34 \mathrm{~cm}$, respectively. Unlike CompuTree or SimpleTree, the STEP algorithm was not able to estimate trunk diameter in the uppermost portions of the trees. Our results show that the STEP algorithm is more adapted to extract DBH and stem diameter automatically from occluded and noisy point clouds. Our study also highlights that SimpleTree and CompuTree require data filtering and results corrections. Conversely, none of these procedures were applied for the implementation of the STEP algorithm.
\end{abstract}

Keywords: forest inventory; stem diameter; diameter at breast height (DBH); terrestrial laser scanner; STEP algorithm; CompuTree; SimpleTree; LiDAR

\section{Introduction}

Long-term preservation of forest resources can only be achieved through accurate forest monitoring and reliable forest inventories, which are accomplished using precise measuring methods. The precise 
measurement of key forest attributes contributes to our understanding of complex ecological processes. Among these attributes, diameter at breast height $(\mathrm{DBH}$, diameter of the trunk at $1.30 \mathrm{~m}$ above the ground-surface) and stem diameter as a function of height are of particular importance for forest inventories.

DBH measurement has become an essential part of a forester's practice and is now a reference variable for the development of allometric models. These models tie DBH to many useful structural variables, especially tree height [1], merchantable or total wood volume [2], biomass [3], and total leaf area [4]. During traditional forest inventories, operators measure DBH using calipers or diameter tapes. However, the operator's visual estimates of the measurement leads to potential errors [5-7]. For example, with calipers, a deviation angle of 2.5 degrees may produce an estimated error of $2 \mathrm{~cm}$ on large trees [6].

Diameter values along the stem, or stem profile, are crucial to enhance wood volume equations and to estimate the useful portion of the stem for wood supply. For most of the stem length, its diameter is more difficult to measure in situ than DBH: logistical issues preclude non-destructive measures. For instance, measuring a standing tree requires that a field operator climb the trunk, limiting the height to which stem diameter measurements can be taken. Consequently, stem diameter cannot be consistently measured as a function of height during forest inventory campaigns with non-destructive methods. Different solutions may be considered for acquiring stem diameter: it can be either measured manually through a destructive protocol or estimated with the help of precise scanning technologies.

Stem profile is measured with destructive protocols that involve felling the trees of interest and cutting the logs into shorter segments. Log circumference can then be measured to obtain a set of diameters along the stem. In the past, field campaigns have been specifically designed to include tree cutting to measure tree stems [8]. However, felling trees is neither an acceptable nor a sustainable practice for most forest inventory plots. Destructive sampling can only be considered for specific purposes: it is incompatible with permanent plot monitoring because of ecological and logistical issues. Consequently, destructive measurements are not a practical solution for regular forestry inventory plots. An efficient solution remains to be developed, without requiring destructive sampling.

Laser scanning technologies become an essential non-destructive solution to monitoring forest environments [9]. LiDAR (Light Detection and Ranging) instruments provide 3D point clouds of their surroundings, containing several tens of millions of points. Two major types of LiDAR instruments have been used to monitor forest environments [10]: airborne laser scanners (ALS) and terrestrial laser scanners (TLS). An ALS is mounted on an aircraft flying over the study area. While it is possible to derive some structural attributes from ALS data, algorithms are mainly designed for stand-level measurements (e.g., aboveground biomass [11]) and for tree attributes that are readily available from above the trees (e.g., tree height [12]; crown area [13]; gaps between crowns [14]). Tree stems are poorly probed with ALS systems because of signal occlusion by the upper canopy. In addition, the ALS point cloud is generally not dense enough to accurately estimate the stem diameter. In contrast, TLS are located on the ground. They emit laser beams in a spherical pattern and convert signal returns into high-density point clouds. TLS can capture stem shape accurately from the lower part of the tree to the top. Yet, TLS data are limited not only by signal occlusion, but also by noise (signal returns not related to a scene object) and non-homogeneous sampling densities. Therefore, there is a need for specialized point-cloud processing algorithms to estimate tree attributes from TLS point clouds [15].

Estimating tree attributes via specialized algorithms offers several advantages: they do not require destructive sampling, and allow to estimate several tree attributes that could not be otherwise measured in the field, such as crown shape and competition [16,17], plant area index [18-20], tree architecture [21,22], and wood fibre attributes [23]. TLS data processing algorithms have thus received much attention in the past few years. Several algorithms have been developed to estimate the structural attributes of trees from TLS point clouds (a review is presented in [15]). In particular, algorithms were proposed for estimating $\mathrm{DBH}$ and stem profile. Most of these algorithms assume that a stem 
cross-section is circular in shape. Thus, they aim to reconstruct stems as a series of circles, or as portions of elliptical surfaces (namely, cylinders and cones).

Circle fitting has successfully retrieved $\mathrm{DBH}$ and stem profile from point clouds. These approaches use clustering methods to select appropriate points within layers and apply either least-squares circle fitting [24-30], a RANSAC algorithm [31], or other methods to retrieve the stem's centre position and radius [32]. More general ellipse fitting has been tested [33-36], but it tends to be sensitive and unstable [37]. Previous studies show radius estimation errors can range from $1 \mathrm{~cm}$ to $3 \mathrm{~cm}$ [38-40]. However, algorithms provide erroneous models when the point cloud is affected by occlusion and noise.

Cylinders have also been used to tackle the issue of circle orientation and the choice of appropriate layers [41-43]. Algorithms have been implemented, such as iterative least-squares cylinder fitting [44] and robust variants using normal information [45,46], or MSAC (M-estimator SAmple and Consensus) [47]. Despite the accuracy of the results, cylinder-based methods are limited by the same factors as circle-based methods.

Tree stem reconstruction as a series of cones was introduced to estimate local tapering of the trunk $[48,49]$, with no major improvement regarding the method's robustness. Other approaches either retrieve a more precise perimeter estimate through b-spline fitting [44], or estimate tree volume by using polygons [50]. These methods result in precise measurements, but require that all of the stems be probed. Therefore, they cannot be applied to single scan data or occluded point clouds.

Another approach to estimating DBH from TLS data identifies circles along the stems using the Hough transform (HT) [38]. As a powerful pattern recognition tool, HT exhibits robustness to both occlusion and noise. It has been successfully used as a prelocalization tool or applied in combination with classical circle fitting [29-31,51,52]. Many solutions for estimating diameter along the stem have been proposed, but occlusion and noise affect most of the algorithms. Consequently, robust methods are still in demand [53].

In an attempt to overcome the limitations of TLS, we developed a new algorithm that is based upon a HT variant called STEP (Snake for Tuboid Extraction from Point cloud) [54]. The STEP algorithm was designed to estimate stem profile in natural forest environments. However, it has not been thoroughly tested on point clouds that have been collected by TLS in forest stands. Stem profile can also be estimated with algorithms that are designed to simulate tree architecture [21,22,55], or with quantitative structural models (QSM) [40,56-58]. These QSM, including SimpleTree software [59], use cylinders to provide a complete description of a tree, including its architecture, stem and branch diameters, and total volume. Yet, their accuracy depends upon the quality of the input point cloud.

There is a need (1) to benchmark these methods to understand their respective advantages and (2) to evaluate their accuracy in various natural forest environments. This study aims to evaluate the STEP algorithm on a diversified set of TLS acquisitions in natural forest environments. As a second goal, we also compared the performance of the STEP algorithm with that of other algorithms. Consequently, our main objective was to assess the accuracy of the STEP algorithm when applied to a range of TLS acquisition protocols for stem profile estimation. This goal was reached through two specific objectives. First, we selected contrasting test sites representing natural forest environments; each site was related to a reliable set of reference stem diameter measurements. This allowed us to compare the estimated values with well-documented measurements that were used as reference values. Second, we selected three stem profile estimation algorithms representing distinct strategies. We compared the reference measurements with the estimation results of (1) the STEP algorithm [54], (2) the advanced cylinder-fitting algorithm that was available in the CompuTree software platform [24], and (3) the SimpleTree QSM algorithm [59].

These three algorithms were all implemented as plug-ins within the CompuTree software platform. The goal of this platform is to regroup TLS point cloud processing algorithms for forest scene analyses. It provides a full development environment based on powerful $\mathrm{C}++$ libraries, and eases the implementation of various methods as plug-ins for comparative and exchange purposes. Benchmarking should provide insights into each of the three algorithms that were selected for the estimation of stem profile. 


\section{Benchmarked Algorithms for Stem Diameter Estimations}

\subsection{STEP Algorithm}

The STEP algorithm was first introduced by Ravaglia et al. [54]. It reconstructs tuboids that are defined as a series of circles with continuous locations, radii and orientations. The STEP algorithm was designed to optimize stem diameter estimation by a two-stage process, while considering the limitations of TLS data (signal occlusion, noise and uneven sampling). The first stage of the algorithm is an efficient variant of HT that has been adapted to circles. This computation is based upon normal directions and produces a discrete $4 \mathrm{D}$ space in which each element represents an occurrence of a circle. A score is assigned to each element, corresponding to the number of points that are located on the corresponding circle. The score of each circle hence reflects the level of confidence of being an accurate model of the data. In the second stage of STEP, growing active contours are used to extract a series of circles with a high score, which correspond to tree stems.

A major limitation of applying classical HT to point clouds is its complexity, which leads to computationally expensive methods. However, HT is robust to occlusion and noise. We introduced normal vectors in a variant of $\mathrm{HT}$ in order to reduce its complexity, while benefiting from its advantages. We used an efficient ray tracing algorithm to compute the $4 \mathrm{D}$ discrete accumulator space (three dimensions for the circle's centre and one dimension for its radius) in linear time [60]. For the first stage of the calculation, each cell of this space corresponds to a 3D circle and is associated with a score reflecting the reliability of the circle.

As a series of contiguous 3D circles, tuboids are represented by continuous curves in the accumulator space. Hence, the second stage of the procedure aims at extracting curves passing through the elements that have a high score. We tackled this issue with growing active contours [61]: we grew a curve in the accumulator space until a growth-stopping criterion (based upon principal component analysis) was reached. This curve was then considered an active contour. Active contours are curves that minimize an energy, including constraints regarding the shape and location of the curve. In our application, we used the energy $E_{g}$ to ensure the geometric accuracy of the active contour $c(u)$ and to force it to pass through high score elements in the accumulator space:

$$
E_{g}=\int\left[E_{i}(c(u))+E_{d}(c(u))\|c \prime(u)\|\right] d u
$$

where $E_{i}(c(u))=\alpha\left\|c_{\prime}(u)\right\|^{2}+\beta\left\|c_{\prime}(u)\right\|^{2}$ is an internal energy term constraining the elasticity and smoothness of the curve, and $E_{d}(c(u))\|c r(u)\|$ is used to compute the linear integral of a data energy term constraining the curve to pass through the elements with a high score:

$$
E_{d}(c(u))=a \frac{H_{m}-H(u)}{H_{M}-H_{m}}+(1-a) \frac{h_{m}-H(u)}{h_{M}-h_{m}}
$$

where $a \in[0,1]$ is a balancing term, $H_{m}$ and $H_{M}$ are respectively the minimum and maximum scores of the accumulator space, and $h_{m}$ and $h_{M}$ are respectively the local minimum and maximum of the accumulator space around $c(u)$. Further details on curve initialization and the optimization procedure that is used to reach the minimum energy are given in [54].

The results of the STEP algorithm depend upon the quality of the normal vector estimates, which remains a challenging task [62]. The main limitations of the STEP algorithm are either the main memory it requires, or its computational time that is due to the use of sparse matrices, depending upon the implementation.

\subsection{Cylinder Fitting Algorithm}

The Office National des Forêts (ONF, French forest office) introduced the CompuTree software platform which includes a stem diameter estimation algorithm based upon the work 
of Ohtmani et al. [24]. It will be referred to as the CompuTree cylinder fitting algorithm (or CCF algorithm) from this point onward. The CCF algorithm was selected because it represents the family of algorithms that use cylinder- or cone-fitting to estimate stem diameter (e.g., [15,48,63]).

The CCF algorithm starts by removing the branches of the trees from the original point cloud with a convolution filter. The algorithm then divides the point cloud into horizontal layers of a fixed thickness. A Euclidean clustering procedure segments each stem individually in these horizontal layers. A cylinder is then fitted to the segments using least-squares optimization. More details regarding this stem diameter estimation process and its evolution of the CCF algorithm since it was first presented can be found on the Computree platform website [64].

The CCF algorithm was designed to require little computational time when applied to a forest plot, while being able to provide accurate estimates of tree location, DBH and stem diameter. It has already demonstrated its efficiency with high stem detection rates [24]. Even though its parameters are intuitive, the CCF algorithm must be parameterized for the point cloud specifications and forest stand characteristics. These parameters include different thresholds that are applicable to 3D convolution filters, Euclidean clustering, and least-squares fitting. Even though the CCF algorithm presently cannot detect horizontal branches, it is adapted to be used with TLS point clouds to estimate DBH and stem diameter in forest inventory applications.

\subsection{SimpleTree}

We selected SimpleTree, which is a QSM algorithm that was developed by Hackenberg et al. [59], as a third algorithm for our comparative study, since it is considered state-of-the-art. We used a version available as a plug-in from the CompuTree platform. A summary of the SimpleTree method follows.

The SimpleTree algorithm provides a tree model (including branches) that is formed by interconnected cylinders that have been generated from circle fittings. It starts modelling the tree from an estimated circle $C_{0}$ at the base of the stem. The centre of $C_{0}$ is used to position a cutting sphere $S_{0}$ with a radius greater than that of $C_{0}$. The intersection between $S_{0}$ and the input point cloud represents a cross-section of the stem. A circle $C_{1}$ is then fitted to the cross-section points and a new sphere $S_{1}$ is generated at the centre of $C_{1}$. The stem is iteratively reconstructed by moving the cutting spheres $S_{i}$ along the fitted circles $C_{i}$. When a cutting sphere intersects several cross-sections, a new branch is detected and reconstructed with the same procedure. The results of the SimpleTree algorithm are available as hierarchical series of cylinders that are derived from the fitted circles $C_{i}$.

The SimpleTree sphere following method runs iteratively until it retrieves the entire tree structure, including the branch diameters and order. It has been enhanced with automatic parameterization, optimization and forestry knowledge, including allometry. However, it requires that the tree be isolated in a single point cloud. We use SimpleTree in the present benchmark to better understand its advantages and limitations over a wide range of natural forest stand acquisitions.

\section{Materials and Methods}

\subsection{Test Sites and Data Sets}

Only a few forest sites matched our field requirements for this benchmarking (i.e., natural environments, with associated reference diameter measurements). We selected three test sites with different, yet accurate, reference measurements to evaluate the selected algorithms. These sites provide diverse structural configurations and different data quality levels. They offer a variety of locations, forest environments, species, data sources, and acquisition protocols.

The three algorithms (STEP, CCF algorithm and SimpleTree) were applied at a tree-level to 81 isolated trees: 24 were from a simulated dataset replicating forest plots in the Province of Newfoundland and Labrador (Canada), 40 were from the Phalsbourg dataset that was collected in the Grand Est region (France), and 17 were from the Bas Saint-Laurent dataset that was collected in the Province of Québec (Canada). The three test sites provided appropriate reference measurements to 
evaluate the three algorithms with a large number of trees in simulated and natural environments. They included both coniferous and deciduous trees, and multiple species. These datasets also provided various limitations with leaf-on scans and various degrees of occlusion and noise. Table 1 presents a summary of the trees that were selected from each dataset for evaluation along with the corresponding scanning protocol.

Table 1. Overview of the data set characteristics and scanning protocol for the three test sites.

\begin{tabular}{cccc}
\hline Study Site & Newfoundland & Phalsbourg & Bas Saint-Laurent \\
\hline Stand type & Coniferous & $\begin{array}{c}\text { Mixed } \\
\text { (mainly deciduous) } \\
\text { Common beech }\end{array}$ & Coniferous \\
Tree species & Black Spruce & Hornbeam & White spruce \\
Number of selected trees & 24 & 40 & 17 \\
Minimum DBH & $5.43 \mathrm{~cm}$ & $10 \mathrm{~cm}$ & $9 \mathrm{~cm}$ \\
Maximum DBH & $19.88 \mathrm{~cm}$ & $70 \mathrm{~cm}$ & $40 \mathrm{~cm}$ \\
TLS scanner & Simulated (PBRT) & Faro Focus 3D & Faro Focus 3D A120 \\
Scanner angular resolution & $0.036^{\circ}$ & $0.036^{\circ}$ & $0.018^{\circ}$ \\
Number of scans per plot & 5 & 4 & 4 \\
\hline
\end{tabular}

\subsubsection{Newfoundland Dataset}

The first dataset is a collection of three simulated forest plots. Each virtual plot was populated with tree models that were obtained from the architectural model L-Architect [22,55]. The model positions each simulated tree according to a tree map location. L-Architect provided the optimal representation of plots for forests in insular Newfoundland (Canada) that are dominated by balsam fir (Abies balsamea (L.) Mill.) and black spruce (Picea mariana (Mill.) BSP). Three virtual forest plots with an area of $900 \mathrm{~m}^{2}$ (square plots with $30 \mathrm{~m}$ edges) were generated and contained 30, 71 and 92 trees, respectively. In all three plots, we applied random tree selection stratified by $10 \mathrm{~cm}$ tree diameter classes. We selected 24 virtual trees with DBH values ranging from $10.86 \mathrm{~cm}$ to $39.76 \mathrm{~cm}$.

TLS acquisitions of these virtual forest plots were simulated using a physically based ray tracing tool with the following scan settings [65]: (1) all scans used an angular resolution of $0.036^{\circ}$ for rays that were emitted throughout the full field-of-view of the instrument, (2) rays were simulated with no divergence, and (3) five scans were co-registered as a quincunx: one scan was taken in the centre of the plot, and four were taken at each extremity of the plot (north, south, east, west). The soil was not modelled by L-Architect. The ray tracing simulations resulted in noiseless point clouds. The trees that were included in the virtual plots had a large number of branches. This generated numerous occlusions of varying importance along the tree stems (Figure 1). These simulated plots were designed to evaluate the effects of occlusion and non-homogeneous density since the point clouds were noiseless and the stems were perfectly cylindrical. The trees were later isolated in the resulting point clouds to apply the stem diameter estimation algorithms at the tree level.

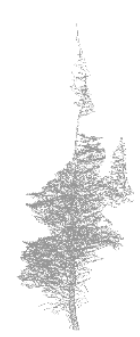

(a)

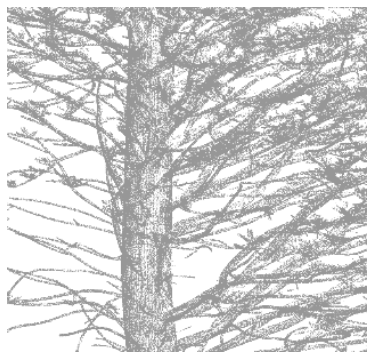

(b)

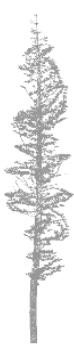

(c)

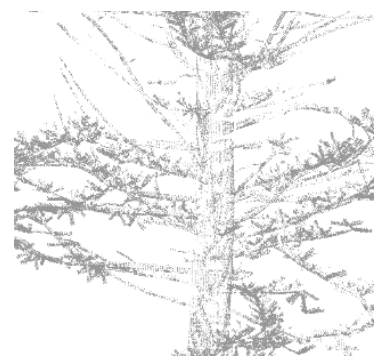

(d)

Figure 1. Examples of simulated point clouds from the Newfoundland data set. Even though the point clouds were noise-free, they suffered from branch occlusion. (a) Isolated tree; (b) Close-up view of branches; (c) Isolated tree; (d) Close-up view of branches. 
Simulated TLS acquisitions of the virtual forest plots are approximations of real scans. However, the L-Architect model provided exact reference values for stem diameter: starting from the tree base, the model specified the stem diameter at every $10 \mathrm{~cm}$ increment.

\subsubsection{Phalsbourg Data Set}

The second dataset contains several TLS point clouds that were acquired in natural forest environments. It was collected during a field inventory campaign in 2013 by the research and development department of the ONF. The study area was located near the city of Phalsbourg, in northeastern France. The selected permanent forest plots (circular plots with a $15 \mathrm{~m}$ radius) included 12 different stands, all of which were dominated by deciduous tree species, with a predominance (in decreasing order) of common beech (Fagus sylvatica L.) and hornbeam (Carpinus betulus L.), Norway spruce (Picea abies (L.) H. Karst), Scots pine (Pinus sylvestris L.), and silver fir (Abies alba Mill.). The plots were scanned during leaf-on season and the DBH of the inventoried trees ranged from $10 \mathrm{~cm}$ to $70 \mathrm{~cm}$. To evaluate the three algorithms, we selected 40 of these trees by using stratified random selection by $10 \mathrm{~cm}$ DBH class bins (Figure 2).

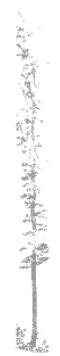

(a)

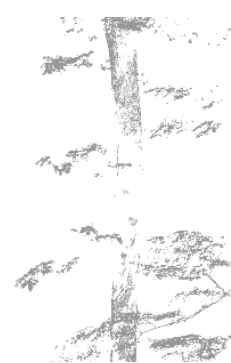

(b)

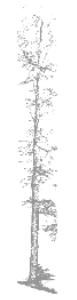

(c)

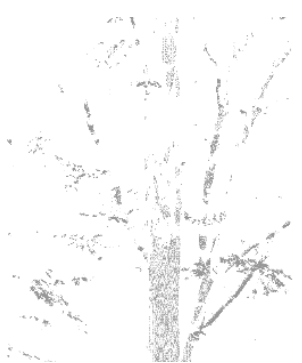

(d)

Figure 2. Examples of point clouds acquired at one of the Phalsbourg test sites. The point clouds exhibit large occluded areas on the stem. (a) Isolated tree; (b) Close-up view of branches; (c) Isolated tree; (d) Close-up view of branches.

Plots were scanned with a Faro Focus $3 \mathrm{D}^{\odot}$ scanner with an angular resolution of $0.036^{\circ}$. Each plot was scanned from a central position as well as three other locations, forming an equilateral triangle $7.5 \mathrm{~m}$ away from the plot's centre. The scan settings induced a non-homogeneous sampling density, and the presence of foliage during the acquisitions resulted in numerous occluded areas. The scans included noise, which was mostly located in the upper part of the trees. This dataset allowed us to assess the effects of occlusion, noise and point density on the performance of the algorithms. The selected trees were isolated into individual point clouds by using a cylindrical extraction of points around their centre.

During the field campaign, inventoried trees were cut down at breast height. Stems were then felled and further cut into $2 \mathrm{~m}$-long logs. The diameter at the end of each log was measured manually twice with a caliper (measurements taken at right angles). Starting from $1.30 \mathrm{~m}$ above the ground surface, manual measurements were available at each $2 \mathrm{~m}$ increment from breast height and served as reference values for stem diameter. With such data, the results of the three algorithms on natural forest sites could be compared to traditional field measurements.

\subsubsection{Bas Saint-Laurent Dataset}

The third dataset groups several TLS point clouds that were acquired in a tree plantation. A research team from the Université du Québec à Rimouski had led a field campaign in the nature reserve of Matane (Québec) during the autumn of 2014. The plantation of white spruce (Picea glauca (Moench) Voss) is located in the balsam fir-yellow birch bioclimatic domain [66]. A $60 \mathrm{~m} \times 15 \mathrm{~m}$ plot was established in the plantation and divided into four $15 \mathrm{~m} \times 15 \mathrm{~m}$ subplots. The resulting subplots included trees with DBHs ranging from $9 \mathrm{~cm}$ to $40 \mathrm{~cm}$. We proceeded with stratified random selection 
with $10 \mathrm{~cm}$ DBH class bins. We selected 17 trees with a DBH ranging from $9 \mathrm{~cm}$ to $37 \mathrm{~cm}$ for the evaluation of the three stem diameter estimation algorithms (Figure 3).

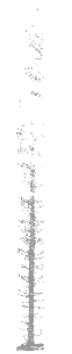

(a)

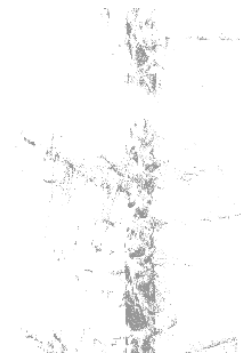

(b)

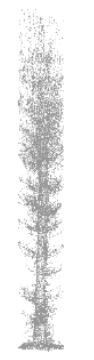

(c)

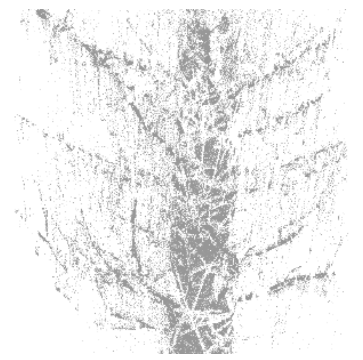

(d)

Figure 3. Examples of point clouds acquired with a terrestrial LiDAR at the Bas Saint-Laurent test sites, mainly composed of white spruce. The point clouds exhibit significant occluded areas and noise on the stem. (a) Isolated tree; (b) Close-up view of branches; (c) Isolated tree; (d) Close-up view of branches.

The plot was scanned using a Faro Focus 3D A120@ scanner with an angular resolution of $0.018^{\circ}$. For each subplot, four scans were acquired at the plot borders ( $7.5 \mathrm{~m}$ away from the subplot centre), yielding a total of 13 scans (Figure 4). The resulting point clouds included abundant occlusions and noise due to dense vegetation (branches and needles). This dataset allowed us to validate the three algorithms in real coniferous plantation sites and to analyze their resilience to occluded and noisy point cloud data. The selected trees were manually isolated into separate point clouds for further processing.

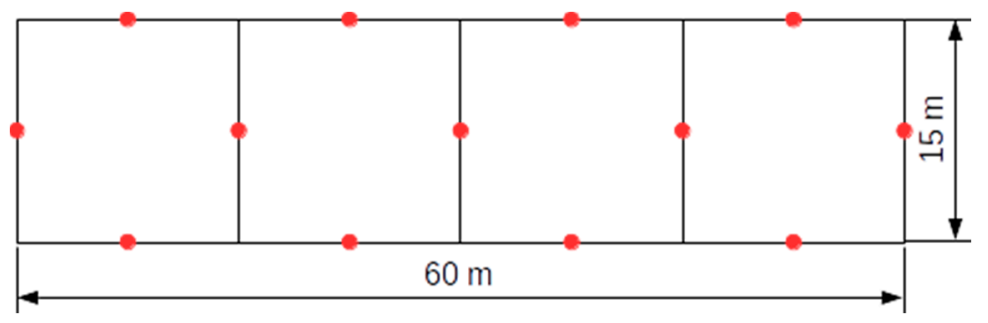

Figure 4. Scanning set-up adopted for the terrestrial LiDAR acquisition at the Bas Saint-Laurent test site. Each of the four sub-plots (black) were scanned from four different positions (red).

Manual measurements were not included in the measurement protocol. The reference stem diameters were obtained from the True-Shape ${ }^{\circledR}$ scanner. The True-Shape ${ }^{\circledR}$ scanner is an accurate log scanning unit used by the forest products industry. It scans stems at a very high-density sampling by using multiple sensors surrounding the fallen stem. This results in an accurate, occlusion-free and noise-free point cloud. During harvest, when trees of interest are felled at their bases, the stems are bucked (pruned of their branches) and the bark is removed. Each stem is then scanned with the True-Shape ${ }^{\circledR}$ unit to obtain a dense representation of the stem cross-sections in $5 \mathrm{~cm}$ increments from where the tree was cut to the tip of the stem. We computed the best-fit circle on the noiseless and occlusion-free point clouds at each recorded height in order to obtain reference measurements of the stem profile. Removal of stem bark incurred an average bias of $1 \mathrm{~cm}$ with respect to field measurements, which was corrected during the analysis.

The three sites that are presented here provided sufficient high-quality material to validate the three algorithms on a large number of trees in simulated environments and under field conditions. The 81 selected trees included both coniferous and deciduous trees, multiple species, and various degrees of noise and occlusion. Thus, these datasets reflect different potential forest inventory sites and protocols. We used them, together with their reference measurements, to evaluate the potential of the three algorithms in forest inventories. While scan setups differed among sites, resulting point clouds were similar enough for comparison. The angular resolutions of the simulated scans (Newfoundland) 
and real scans (Phalsbourg) were identical $\left(0.036^{\circ}\right)$. The setup of sensor positions in multi-view scanning were comparable for all sites.

\subsection{Comparison}

Let us now give an overview of the four step-procedure we used to compare the three selected algorithms. For the first step, we parameterized the algorithms with an equal number of randomly selected trees from each test site. For the second step, we ran the three algorithms on all the selected trees. When necessary, we eliminated branches reconstructions during a post-processing analysis: we manually discarded cylinders fitted on erroneous parts of the point clouds. For the third step, we transformed the set of reference measurements and estimations into continuous diameter functions using linear interpolation. Finally, for the fourth step, we chose an error metric to compare the results of each algorithm against the reference values.

For the first step of our methodology, we parameterized the three algorithms to achieve accurate results for each study site. We used an iterative trial-and-error experiment to find the optimal parameter set for each algorithm. We summarized the most important parameters for each algorithm. The two major parameters to be established for the STEP algorithm were the size of the growing search cone and the value of the growth-stopping criterion. The most important parameters of the CCF chain were those of the convolution filter, the threshold in the Euclidean clustering, and the RMSE threshold for accepting or discarding a fitted cylinder. As for the SimpleTree approach, outlier removal and the voxel grid filter were the main processes that needed to be parameterized: the intrinsic parameters of the SimpleTree algorithm are automatically set.

In the second step, we applied the algorithms to all trees, using the previously selected set of parameters. Unlike STEP, results of the CCF and SimpleTree algorithms included some estimates of branch diameters. The CCF algorithm produced some cylinders corresponding to branches. Simpletree reconstructed entire trees, labeling cylinders as branches or as the trunk. Yet, the labels contained some errors. Only stem diameter estimation was required for the comparison of the algorithms. Therefore, we removed the branches based upon visual assessment. We subsequently inspected the output of the algorithms and manually filtered erroneous cylinders.

As the third step of our methodology, we transformed the discrete diameter measurements into continuous stem profile functions to compare the results. Allometric relationships are a valid option, which was not explored in this study. There are many taper models from which to choose. In our study, too few individuals were available to accurately parameterize these models. We preferred using linear interpolations instead of allometric models. This choice was based upon simplicity and avoided complex issues that are related to allometric modelling. The distance between each reference measurement along the stems is small compared to tree heights $(10 \mathrm{~cm}$ height increment for the simulated forest plots, $2 \mathrm{~m}$ for the Phalsbourg dataset, and $5 \mathrm{~cm}$ for the Bas Saint-Laurent trees, see Figure 5). Therefore, linear interpolation is a pertinent approach, given that it can be viewed as a first-order limited development of the usually low curvature of taper models. For each tree, continuous reference diameter measurements were obtained with piecewise linear interpolation of the reference diameters (edges between black dots in Figure 5). We applied the same linear interpolation to the output of the three algorithms.

As the final step of our methodology, we selected metrics to assess stem diameter estimation error. To do so, both reference and estimated continuous diameter functions were regularly resampled in $2 \mathrm{~cm}$ increments. For each sampled height $h_{i}$, the absolute error $E_{\mathrm{abs}}\left(h_{i}\right)$ was defined as the difference between the absolute values of the reference diameter measurement $d_{r}\left(h_{i}\right)$ and the estimated diameter $d_{e}\left(h_{i}\right)$ :

$$
E_{\mathrm{abs}}\left(h_{i}\right)=\left|d_{r}\left(h_{i}\right)-d_{\mathcal{e}}\left(h_{i}\right)\right|
$$


We also considered the relative error $E_{r e l}\left(h_{i}\right)$ which was defined as a ratio of the reference measurements:

$$
E_{r e l}\left(h_{i}\right)=\frac{\left|d_{r}\left(h_{i}\right)-d_{e}\left(h_{i}\right)\right|}{d_{r}\left(h_{i}\right)}
$$

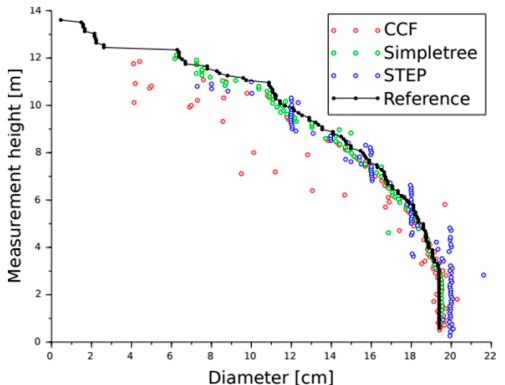

(a)

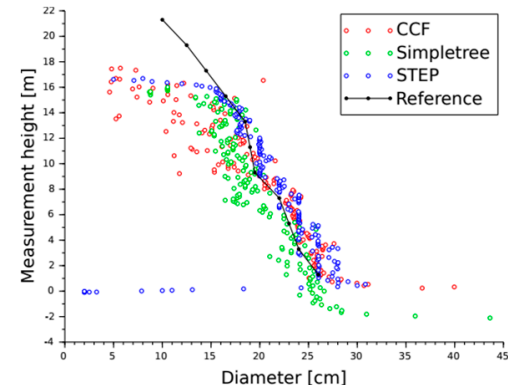

(b)

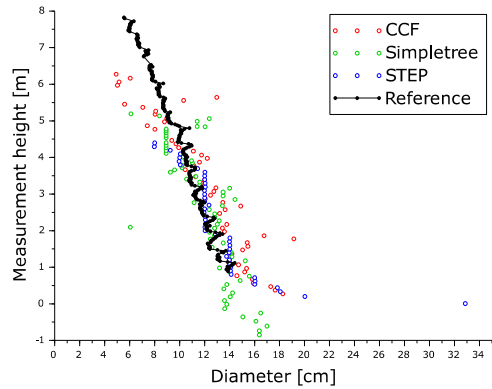

(c)

Figure 5. Example of the piecewise linear interpolation of reference measurements on three example trees from: (a) Newfoundland, (b) Phalsbourg, and (c) Bas Saint-Laurent test sites.

\section{Results}

We computed the error on $\mathrm{DBH}$ and stem profile for each dataset and each algorithm. We computed the stem profile error function globally and at tree-level, together with its minimum, maximum, mean, standard deviation and RMSE. In addition to these general statistics, we considered the distribution of the diameter error (1) per tree and (2) per $20 \mathrm{~cm}$ height interval. Further, we recorded the maximum reconstruction height for each algorithm. This height is computed as the height above the ground of the highest diameter that was estimated by each algorithm. The point clouds did not cover the entire upper part of the trees because of foliage occlusion. It was difficult even for operators to provide an objective height at which stems were sufficiently visible to be reconstructed. Therefore, we only provide maximum reconstruction heights as information regarding the ability of algorithms to reconstruct the upper parts of stems.

Details regarding the maximum height that could be measured for each tree with each method are provided in Table 2. The SimpleTree algorithm reconstructed the tree stems of the Newfoundland site up to the visible treetops, but the reconstructed stems that were obtained with the two other methods excluded the uppermost portions of the trees. For the Phalsbourg dataset, the three algorithms reconstructed the stems up to similar heights with differences of $0.5 \mathrm{~m}$ at most. Finally, for the Bas Saint-Laurent site, the STEP algorithm reconstructed the stems to a lower height when compared with CCF and SimpleTree.

Table 2. Reconstruction height with the three algorithms.

\begin{tabular}{ccccc}
\hline $\begin{array}{c}\text { Reconstruction } \\
\text { Height [m] }\end{array}$ & Algorithm & Newfoundland Site & Phalsbourg Site & Bas Saint-Laurent Site \\
\hline & CCF & 9.35 & 11.02 & 5.95 \\
Mean & SimpleTree & 10.22 & 11.21 & 6.37 \\
& STEP & 8.80 & 10.66 & 4.88 \\
Std deviation & CCF & 3.29 & 4.17 & 0.92 \\
& SimpleTree & 3.22 & 3.61 & 1.09 \\
& STEP & 2.09 & 3.58 & 1.42 \\
\hline
\end{tabular}

The accuracy of DBH estimation for each algorithm is illustrated in Figure 6. The three selected algorithms achieved $0.25 \mathrm{~cm}$ to $3 \mathrm{~cm}$ accuracy regarding tree DBH estimation. With fewer outliers, higher $\mathrm{R}^{2}$, and lower RSME, the STEP algorithm tended to produce better results than the CCF and SimpleTree algorithms for the Phalsbourg and Bas Saint-Laurent datasets. 

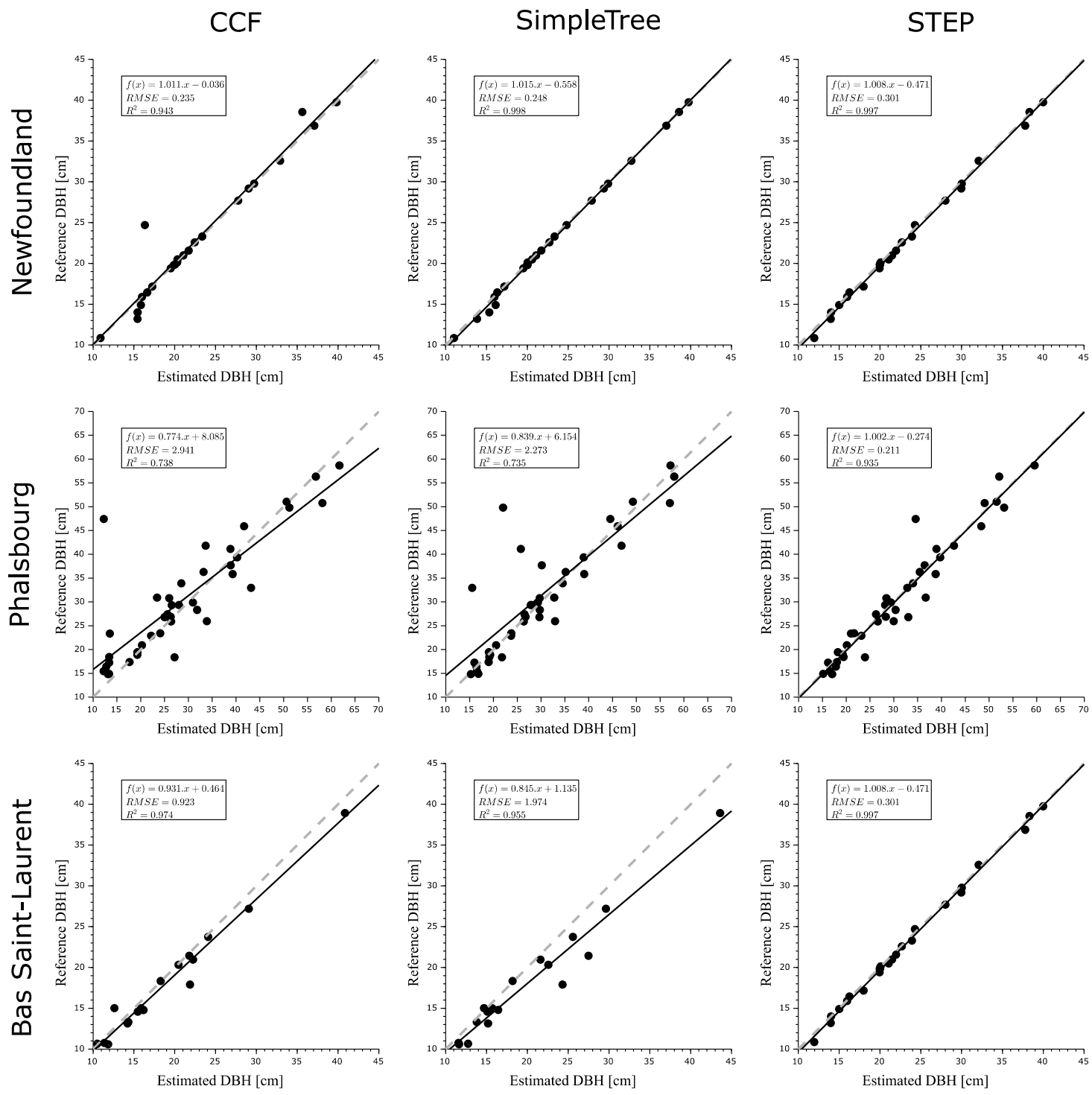

Figure 6. Diameter at breast height $(\mathrm{DBH})$ estimation error using the three selected algorithms for each site. The grey dotted line is the 1:1 line.

Stem diameter errors (averaged per tree and by height) between the reference and estimated values are shown for each study site in Figure 7 and Table 3. They show that the STEP method produces lower mean errors for the Phalsboug and Bas Saint-Laurent sites than with the other two algorithms. With average respective errors per tree of $2.28 \mathrm{~cm}$ and $1.5 \mathrm{~cm}$ for the Phalsbourg and Bas Saint-Laurent sites, the STEP algorithm provides better results than the CCF algorithm and the SimpleTree algorithm. Yet, the SimpleTree and STEP algorithms achieved similar errors on the Newfoundland dataset $1.03 \mathrm{~cm}$ for SimpleTree; $1.11 \mathrm{~cm}$ for STEP), which were lower than what the CCF algorithm provided. On the Phalsbourg and Bas Saint-Laurent sites, the STEP algorithm also performed better than the CCF and SimpleTree algorithms in the lower portions of the trees. Results also show that the error recorded for the three algorithms tended to increase with measurement height when applied to real datasets. 


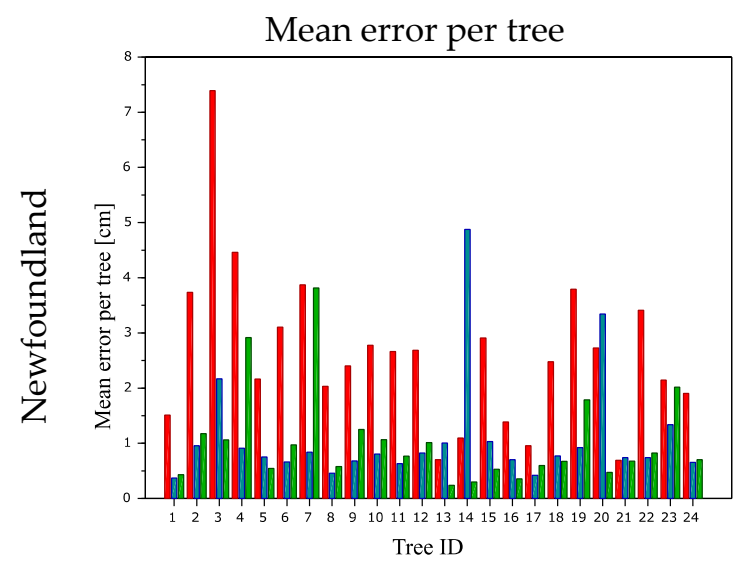

(a)

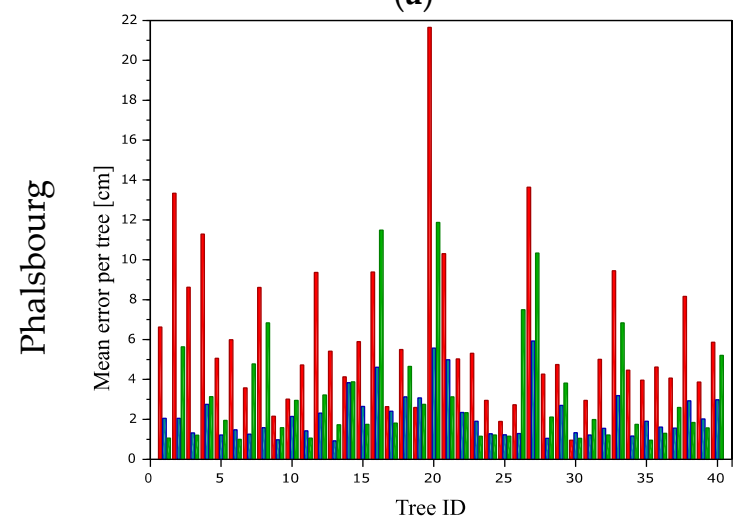

(c)

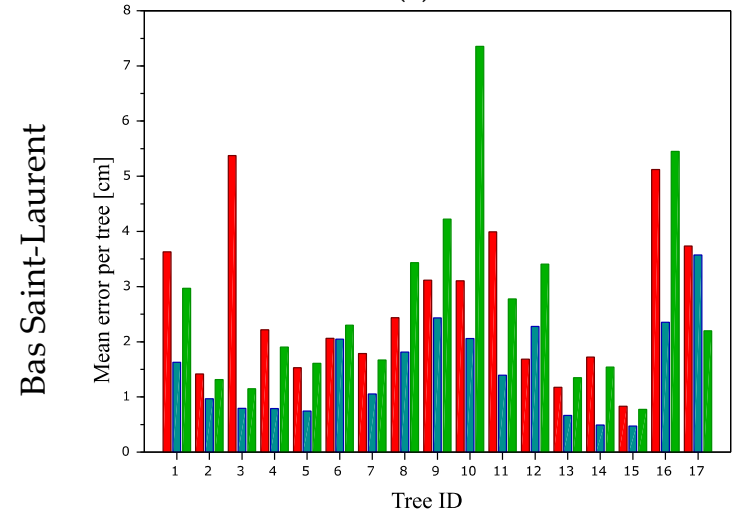

(e)

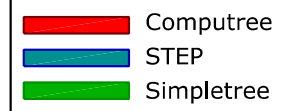

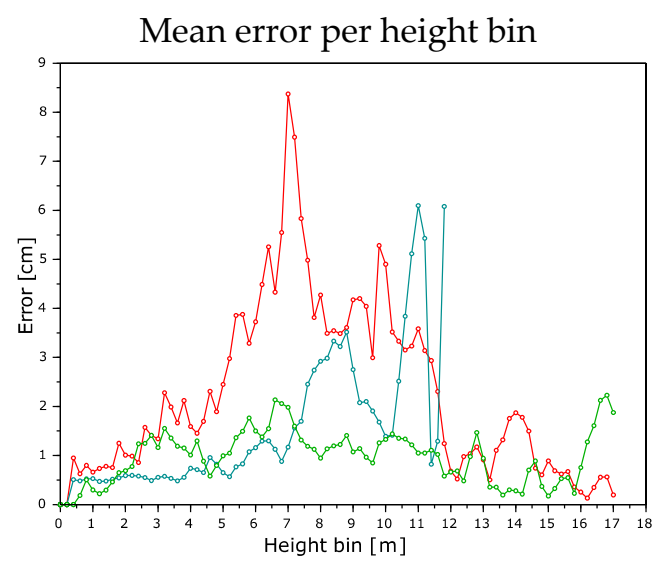

(b)

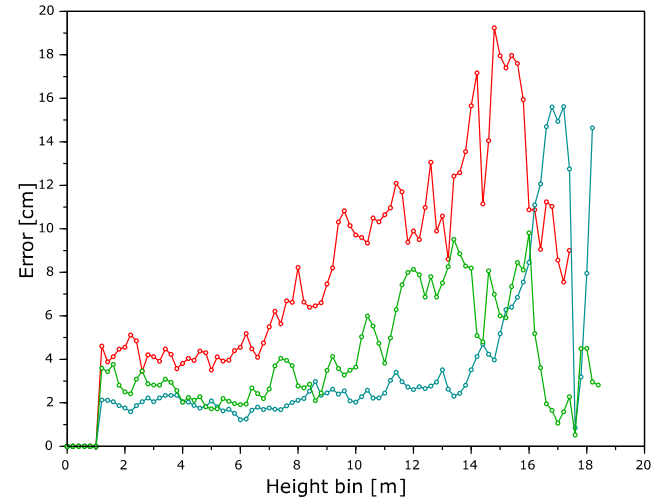

(d)

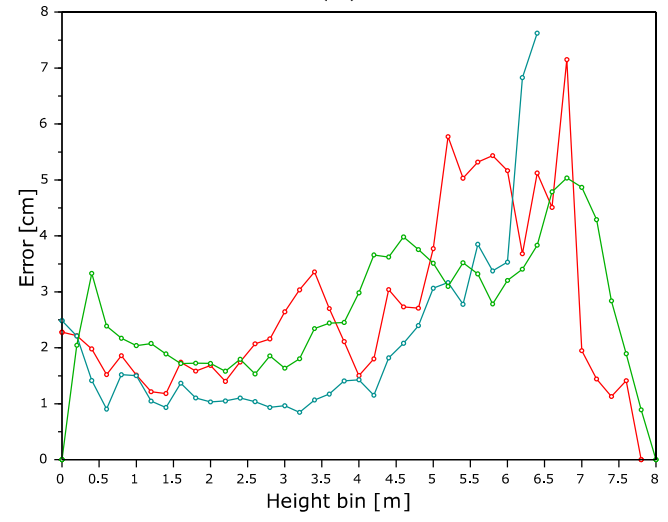

(f)

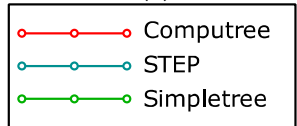

Figure 7. Stem profile estimation error for each site and each algorithm. The left figures (a, c, and e) show the mean profile error for each individual tree (labelled by tree ID) and each algorithm (red, green and blue). The right figures (b, d, and $\mathbf{f}$ ) show the mean profile error per $20 \mathrm{~cm}$ bin for each site and each algorithm. 
Table 3. Results of the stem diameter estimation with the three selected algorithms.

\begin{tabular}{|c|c|c|c|c|c|c|c|}
\hline \multirow{2}{*}{$\begin{array}{l}\text { Diameter Error } \\
\text { per Tree }[\mathrm{cm}]\end{array}$} & \multirow[t]{2}{*}{ Algorithm } & \multicolumn{2}{|c|}{$\begin{array}{l}\text { Newfoundland Site } \\
\text { (24 Selected Trees) }\end{array}$} & \multicolumn{2}{|c|}{$\begin{array}{l}\text { Phalsbourg Site } \\
\text { (40 Selected Trees) }\end{array}$} & \multicolumn{2}{|c|}{$\begin{array}{c}\text { Bas Saint-Laurent Site } \\
\text { (17 Selected Trees) }\end{array}$} \\
\hline & & Abs [cm] & $\operatorname{Rel}[\%]$ & Abs [cm] & $\operatorname{Rel}[\%]$ & Abs $[\mathrm{cm}]$ & $\operatorname{Rel}[\%]$ \\
\hline \multirow{3}{*}{ Minimum } & CCF & 0.69 & 5.82 & 0.96 & 5.98 & 0.83 & 18.92 \\
\hline & SimpleTree & 0.24 & 1.61 & 0.95 & 3.82 & 0.77 & 8.13 \\
\hline & STEP & 0.37 & 2.00 & 0.93 & 3.11 & 0.47 & 4.69 \\
\hline \multirow{3}{*}{ Global average } & CCF & 2.61 & 16.01 & 6.25 & 23.61 & 2.60 & 18.52 \\
\hline & SimpleTree & 1.10 & 6.75 & 3.41 & 12.54 & 2.64 & 16.94 \\
\hline & STEP & 1.13 & 11.31 & 2.34 & 9.34 & 1.52 & 8.95 \\
\hline \multirow{4}{*}{$\begin{array}{c}\text { Global } \\
\text { Std deviation }\end{array}$} & CCF & 3.94 & 28.03 & 7.47 & 24.27 & 3.44 & 28.12 \\
\hline & SimpleTree & 2.51 & 11.26 & 5.32 & 16.59 & 2.74 & 13.95 \\
\hline & STEP & 2.61 & 63.68 & 3.09 & 12.88 & 1.83 & 10.57 \\
\hline & CCF & 4.72 & - & 9.74 & - & 4.31 & - \\
\hline \multirow[t]{2}{*}{ Global RMSE } & SimpleTree & 2.74 & - & 6.32 & - & 3.80 & - \\
\hline & STEP & 2.84 & - & 3.87 & - & 2.38 & - \\
\hline \multirow{3}{*}{$\begin{array}{l}\text { Average error at } \\
\text { tree-level }\end{array}$} & CCF & 2.62 & 15.78 & 6.1 & 24.71 & 2.64 & 18.92 \\
\hline & SimpleTree & 1.03 & 6.55 & 3.34 & 12.72 & 2.67 & 16.85 \\
\hline & STEP & 1.11 & 10.40 & 2.28 & 9.76 & 1.5 & 9.31 \\
\hline \multirow{3}{*}{$\begin{array}{l}\text { Std deviation } \\
\text { at tree-level }\end{array}$} & CCF & 1.44 & 6.97 & 3.96 & 11.44 & 1.35 & 12.31 \\
\hline & SimpleTree & 0.85 & 4.11 & 2.88 & 7.10 & 1.72 & 5.01 \\
\hline & STEP & 1.01 & 19.18 & 1.25 & 4.11 & 0.87 & 4.82 \\
\hline \multirow{3}{*}{$\begin{array}{c}\text { Average RMSE at } \\
\text { tree-level }\end{array}$} & CCF & 4.38 & - & 8.09 & - & 3.85 & - \\
\hline & SimpleTree & 1.86 & - & 4.73 & - & 3.29 & - \\
\hline & STEP & 1.79 & - & 3.13 & - & 2.03 & - \\
\hline \multirow{3}{*}{ Maximum } & CCF & 7.39 & 32.05 & 21.65 & 62.30 & 5.37 & 60.81 \\
\hline & SimpleTree & 3.81 & 15.12 & 11.87 & 28.97 & 7.35 & 27.27 \\
\hline & STEP & 4.88 & 89.21 & 5.93 & 19.15 & 3.57 & 24.25 \\
\hline
\end{tabular}

\section{Discussion}

This study aimed at expanding our current capabilities to estimate stem profile. We tested the STEP algorithm in natural forest environments and provided quantitative results on forest scenes instead of the visual assessments in [54]. Our results illustrated how the STEP algorithm is resilient to occlusion and noise in the point cloud. The RMSE values obtained for DBH estimation by the three algorithms (Figure 6) were improved when compared with previous studies. In addition, of the three algorithms, the STEP algorithm stands out with lower RMSE and higher $\mathrm{R}^{2}$ than CCF and Simpletree: RMSE on DBH estimates ranged from 0.21 to $0.56 \mathrm{~cm}$ for STEP, from 0.24 to $2.94 \mathrm{~cm}$ for $\mathrm{CCF}$, and from 0.25 to $2.28 \mathrm{~cm}$ for Simpletree. In comparison, several other studies propose a range of RMSE for DBH estimates: 0.7 to $7 \mathrm{~cm}$ [15], 2 to $4.2 \mathrm{~cm}$ [31], 0.53 to $0.73 \mathrm{~cm} \mathrm{[67],} 2.39 \mathrm{~cm}$ [68], and $1.27 \mathrm{~cm}$ [69]. In addition to DBH estimation, algorithms were compared for their ability to estimate the tree rate detection [24,70], or above ground biomass [56,57,71,72]. For example, Ohtmani et al. [24] evaluated the CCF algorithm on stem detection rate and DBH, and Hackenberg et al. $[40,58]$ evaluated SimpleTree on DBH and aboveground biomass estimation. Evaluating the algorithms for stem profiles is more challenging than estimating DBH or aboveground biomass, particularly for acquiring reliable reference datasets.

Our results concur with the few studies available on the estimation of the stem profile. STEP estimated stem profile with mean RMSE per tree ranging from 1.79 to $2.03 \mathrm{~cm}$. These results are better than those from Maas et al. [73] with an RMSE of $4.7 \mathrm{~cm}$ obtained on a single tree. Henning et al. [27] obtained an average diameter error of $0.2 \mathrm{~cm}$ (with a standard deviation of $2.1 \mathrm{~cm}$ ) for their estimation of stem profiles, but it was based on tenth-percentiles of all fitted circles within each vertical bins of $1 \mathrm{~m}$. More recently, Liang et al. [46] obtained an RMSE on stem profile of $1.13 \mathrm{~cm}$. This low value can be explained by two aspects related to their experiment: the plot scanned from 7 positions (hence diminishing occlusions) and the stem profiles were filtered (through a smoothing procedure). These studies and our work highlight the difficulty to estimate stem profiles within a compact crown where occlusion occurs and sampling density varies greatly [15,31]. Consequently, 
stem profile estimation error increases with height in the crown (Figure 7). In order to overcome constraints due to signal occlusion, several studies tested their algorithms in relatively favorable conditions. They selected leafless or isolated trees, or either scanned each target tree from 3 to 4 scan positions $[8,40,56,57,71]$ and up to 7 or 8 scan positions $[46,59]$. In contrast, our evaluation is based on 81 trees from point clouds collected using a practical protocol adapted for natural forest stand involving 4 to 5 scans per plot [23]. Such configuration generates point clouds with a higher signal occlusion and variable sampling density compared with experiments tailored for individual tree involving multiple scans per tree. Many methods try to overcome noise and occlusion by applying filters before the geometrical reconstruction of stems $[59,67,71]$. The main goal of such filters is to remove noise from the data. Some methods also filter the estimated stem profile with smoothing or using allometry to correct unrealistic results $[46,58]$. Similarly, our tests with the CCF algorithm and SimpleTree required manual processing: cylinders generated had to be manually processed to keep only those visually associated with the tree stem. The fully automated STEP algorithm instead used unfiltered input data and unaltered results. This configuration was adopted to test STEP ability to deal with signal occlusion and variable sampling. Overall, the results obtained from STEP are generally equivalent or better than those obtained from the CCF algorithm and SimpleTree (Figure 7 and Table 3). This is a strong argument to believe that the complexity of the STEP algorithm, in particular the adoption of the computationally intensive 4D Hough transform, is an effective solution for forest inventories.

We faced several limitations during this study. The foremost limitation is the availability of reference datasets with reliable in situ measurements of the diameter along the entire stem. Adopting a virtual scene has the advantage of testing methods against an absolute reference $[40,56,74,75]$. However, simulated point clouds do not reach the complexity of in situ scans of forest stands. Noise in the point cloud and complete architecture of natural stands are important factors affecting the accuracy of the estimates. Hence a comprehensive evaluation therefore requires non-simulated datasets. While DBH can be easily measured in situ, stem diameter with height is rarely measured manually. That is why most existing studies focused on DBH estimation instead of stem profile. We circumvented this limitation by using two references datasets using the TruShape scanner (Bas St-Laurent site) and in situ diameter measurement after felling the trees (Phalsbourg site). As a second limitation, there is a wide array of processing and parameterization options. For instance, manual cylinder adjustment was applied to the CCF algorithm and SimpleTree but not on the STEP algorithm. These different options make algorithms comparison difficult. Regardless, the estimated stem profile from the three methods were very close to what was measured in situ. Considering that stem profile is not easily measured in situ, the TLS-driven algorithms are an important step towards improving forest inventories. A third limitation was the ability of the algorithms to deal with signal occlusion, variable sampling and noise within the point cloud. This limitation has been raised as one of the major constraints for algorithm development $[15,31]$. Considering that STEP automatically processed unfiltered datasets, it stands out favorably in our analysis with lower RMSE, when compared with the CCF algorithm and SingleTree, for its ability to deal with this limitation.

From our tests and results, we suggest three ways to improve the current stem profile estimation methods. First, procedures can be found in the literature to ease point cloud processing such as point filtering [76], normal vector assessment [62,76,77], or wood-foliage separation to name a few [78,79]. They can reduce noise or the number of irrelevant objects (e.g., foliage) present in point clouds. They can also reduce point density to improve computing time. These procedures could be tested to identify those relevant and suggest a unified framework prior to applying profile estimation algorithms. Such framework would facilitate algorithms comparison on a common base and ease algorithm development. In addition, proposing sensitivity analyses can guide users on how to parameterize the algorithms, and guide the developers towards auto-parametrizing algorithms, which is a current limitation. For example, SimpleTree and Kunz et al. [71] integrated what they called, "brute-force auto-parameterization", which is not the case for the CCF, STEP and most algorithms. A second potential improvement is to enhance the stem profile estimation in the upper part of the tree crown. 
Table 2 identified how STEP can reconstruct stem profiles up to a height $0.5 \mathrm{~m}$ to $1.1 \mathrm{~m}$, and $1.5 \mathrm{~m}$ lower than what was available from the CCF algorithm and SimpleTree respectively. This current limitation of the STEP algorithm is associated with its growth stopping criterion, which needs to be made adaptive according to position in the crown and point cloud conditions. A third potential improvement relates to the TLS acquisition procedures. Considering that occlusions and sampling density affect the algorithms, TLS acquisition protocols can be set up to reduce occlusions. This implies more scans at different positions and results in larger point clouds. Yet, managing huge point clouds is also a serious problem, therefore, a compromise needs to be found. Stem reconstruction from point clouds does not need to be restricted to TLS datasets. Mobile sensors are becoming available and affordable. Similarly, LiDARs mounted on Unmanned Airborne Vehicles (UAVs) acquire coarser point clouds than TLS but they provide an improved visibility of the upper part of the trees. Therefore, UAV LiDAR data may prove to be appropriate for upper stem diameter estimations. However, testing the available algorithms on such data remains to be explored.

\section{Conclusions}

We validated the STEP algorithm with a large set of trees that were extracted from TLS acquisitions in forest environments under various conditions. Overall, the results show that accuracy of the STEP method is comparable to that of manual measurements with conventional instruments, with mean errors ranging from $1 \mathrm{~cm}$ to $2 \mathrm{~cm}$. We also compared the STEP algorithm to two other approaches that are dedicated to stem diameter estimation. The STEP algorithm was more effective when dealing with occluded and noisy point clouds than were the other two methods. We highlighted differences in the processing chains of each algorithm; we believe that adoption of common pre- and post-processing protocols could further improve the current results. The increasing robustness of automatic measurement algorithms pushes the limits of LiDAR-aided forest inventories. Yet, new instruments and technologies (e.g., drone LiDAR and drone photogrammetry) widen the possible application of our present solutions to other operational contexts.

Author Contributions: Conceptualization, J.R., R.A.F. and A.B.; Data Curation, U.R., J.F.C. and A.P.; Formal Analysis, J.R.; Funding Acquisition, R.A.F.; Investigation, J.R.; Methodology, J.R., R.A.F. and A.B.; Project Administration, R.A.F., J.F.C.; Software, J.R.; Supervision, R.A.F. and J.R.; Validation, J.R., J.F.C., R.A.F. and A.B.; Visualization, J.R. and R.A.F.; Writing-Original Draft, J.R., R.A.F. and A.B.; Writing一Review \& Editing, J.R., R.A.F., C.V., J.F.C., A.B. and A.P.

Funding: This research was supported by research grants from FRQNT (Fonds de recherche du Québec-Nature et technologies) and NSERC (Natural Sciences and Engineering Research Council of Canada). The FRQNT project was entitled "Amélioration de l'exactitude et du contenu en information structurale de l'inventaire forestier" (FRQNT file: FT104357, Grantee: J. Bégin, SFB, Ulaval). NSERC funding was linked with the AWARE project (NSERC File: CRDPJ 462973-14, Grantee: N.C. Coops, FRM, UBC), in collaboration with the Canadian Wood Fiber Centre (CWFC), FP-Innovations, and the forest products company Corner Brook Pulp and Paper Ltd. (Corner Brook, NL, Canada) forest company. Cédric Vega is supported by a public grant overseen by the French National Research Agency (ANR) as part of the "Investissements d'Avenir" program (ANR-11-LABX-0002-01, Lab of Excellence ARBRE).

Acknowledgments: We thank Jan Hackenberg of the DIABOLO (Distributed, Integrated and Harmonised Forest Information for Bioeconomy Outlooks) project for his support with the Computree version of SimpleTree.

Conflicts of Interest: The authors declare no conflict of interest. The funders had no role in the design of the study; in the collection, analyses, or interpretation of data; in the writing of the manuscript, or in the decision to publish the results.

\section{References}

1. Feldpausch, T.R.; Banin, L.; Phillips, O.; Baker, T.R.; Lewis, S.L.; Quesada, C.A.; Affum-Baffoe, K.; Arets, E.J.M.M.; Berry, N.J.; Bird, M.; et al. Height-diameter allometry of tropical forest trees. Biogeosciences 2011, 8, 1081-1106. [CrossRef]

2. Fortin, M.; Schneider, R.; Saucier, J.-P. Volume and Error Variance Estimation Using Integrated Stem Taper Models. For. Sci. 2012, 59, 345-358. [CrossRef] 
3. Wulder, M.A.; White, J.C.; Fournier, R.A.; Luther, J.E.; Magnussen, S. Spatially Explicit Large Area Biomass Estimation: Three Approaches Using Forest Inventory and Remotely Sensed Imagery in a GIS. Sensors 2008, 8, 529-560. [CrossRef] [PubMed]

4. Black, K.; Reidy, B.; Bolger, T.; Tobin, B.; Osborne, B.; Nieuwenhuis, M. Assessment of allometric algorithms for estimating leaf biomass, leaf area index and litter fall in different-aged Sitka spruce forests. Forestry 2006, 79, 453-465.

5. Köhl, M.; Magnussen, S.; Marchetti, M. Sampling Methods, Remote Sensing and GIS Multiresource Forest Inventory; Springer Science \& Business Media: Berlin, Germany, 2006.

6. van Laar, A.; Akca, A. Forest Mensuration; Springer Science \& Business Media: Berlin, Germany, 2007.

7. Avery, T.E.; Burkhart, H.E. Forest Measurements; McGraw-Hill: New York, NY, USA, 2001.

8. Dassot, M.; Colin, A.; Santenoise, P.; Fournier, M.; Constant, T. Terrestrial laser scanning for measuring the solid wood volume, including branches, of adult standing trees in the forest environment. Comput. Electron. Agric. 2012, 89, 86-93. [CrossRef]

9. Dassot, M.; Constant, T.; Fournier, M. The use of terrestrial LiDAR technology in forest science: Application fields, benefits and challenges. Ann. For. Sci. 2011, 68, 959-974. [CrossRef]

10. Vosselman, G.; Maas, H.-G. Airborne and Terrestrial Laser Scanning; CRC Press: Boca Raton, FL, USA, 2010; p. 318.

11. Bouvier, M.; Durrieu, S.; Fournier, R.; Saint-Geours, N.; Vincent, G.; Guyon, D.; Grau, E.; Hérault, B. Influence of sampling design parameters on biomass predictions derived from airborne lidar data. In Proceedings of the SilviLaser 2015, La Grande Motte, France, 28-30 September 2015; pp. 137-139.

12. Falkowski, M.J.; Smith, A.M.; Hudak, A.T.; E Gessler, P.; A Vierling, L.; Crookston, N.L. Automated estimation of individual conifer tree height and crown diameter via two-dimensional spatial wavelet analysis of lidar data. Can. J. Remote Sens. 2006, 32, 153-161. [CrossRef]

13. Næsset, E. Predicting forest stand characteristics with airborne scanning laser using a practical two-stage procedure and field data. Remote Sens. Environ. 2002, 80, 88-99. [CrossRef]

14. Vepakomma, U.; St-Onge, B.; Kneeshaw, D. Spatially explicit characterization of boreal forest gap dynamics using multi-temporal lidar data. Remote Sens. Environ. 2008, 112, 2326-2340. [CrossRef]

15. Liang, X.; Kankare, V.; Hyyppä, J.; Wang, Y.; Kukko, A.; Haggrén, H.; Yu, X.; Kaartinen, H.; Jaakkola, A.; Guan, F.; et al. Terrestrial laser scanning in forest inventories. ISPRS J. Photogramm. Remote Sens. 2016, 115, 63-77. [CrossRef]

16. Metz, J.; Seidel, D.; Schall, P.; Scheffer, D.; Schulze, E.-D.; Ammer, C. Crown modeling by terrestrial laser scanning as an approach to assess the effect of aboveground intra-and interspecific competition on tree growth. For. Ecol. Manag. 2013, 310, 275-288. [CrossRef]

17. Olivier, M.-D.; Robert, S.; Fournier, R.A. Response of sugar maple (Acer saccharum, Marsh.) tree crown structure to competition in pure versus mixed stands. For. Ecol. Manag. 2016, 374, 20-32. [CrossRef]

18. Durrieu, S.; Allouis, T.; Fournier, R.; Véga, C.; Albrech, L. Spatial quantification of vegetation density from terrestrial laser scanner data for characterization of 3D forest structure at plot level. In Proceedings of the SilviLaser 2008, Edinburgh, UK, 17-19 September 2008; pp. 325-334.

19. Béland, M.; Widlowski, J.-L.; Fournier, R.A.; Côté, J.-F.; Verstraete, M.M. Estimating leaf area distribution in savanna trees from terrestrial LiDAR measurements. Agric. For. Meteorol. 2011, 151, 1252-1266. [CrossRef]

20. Hosoi, F.; Nakai, Y.; Omasa, K. Voxel tree modeling for estimating leaf area density and woody material volume using 3-D LIDAR data. ISPRS Ann. Photogramm. Remote Sens. Spat. Inf. Sci. 2013, 5, 115-120. [CrossRef]

21. Côté, J.-F.; Fournier, R.A.; Egli, R. An architectural model of trees to estimate forest structural attributes using terrestrial LiDAR. Environ. Model. Softw. 2011, 26, 761-777. [CrossRef]

22. Côté, J.-F.; Fournier, R.A.; Frazer, G.W.; Niemann, K.O. A fine-scale architectural model of trees to enhance LiDAR-derived measurements of forest canopy structure. Agric. For. Meteorol. 2012, 166, 72-85. [CrossRef]

23. Blanchette, D.; Fournier, R.A.; Luther, J.E.; Côté, J.-F. Predicting wood fiber attributes using local-scale metrics from terrestrial LiDAR data: A case study of Newfoundland conifer species. For. Ecol. Manag. 2015, 347, 116-129. [CrossRef]

24. Othmani, A.; Piboule, A.; Krebs, M.; Stolz, C. Towards automated and operational forest inventories with T-Lidar. In Proceedings of the SilviLaser 2011, Hobart, Australia, 16-20 October 2011; pp. 1-9. 
25. Kuusk, A.; Lang, M.; Märdla, S.; Pisek, J. Tree stems from terrestrial laser scanner measurements. For. Stud. 2015, 63, 44-55. [CrossRef]

26. Brunner, A.; Gizachew, B. Rapid detection of stand density, tree positions, and tree diameter with a 2D terrestrial laser scanner. Eur. J. For. Res. 2014, 133, 819-831. [CrossRef]

27. Henning, J.G.; Radtke, P.J. Detailed stem measurements of standing trees from ground-based scanning lidar. For. Sci. 2006, 52, 67-80.

28. Bienert, A.; Scheller, S.; Keane, E.; Mullooly, G.; Mohan, F. Application of Terrestrial Laser Scanners For The Determination Of Forest Inventory Parameters. Int. Arch. Photogramm. Remote Sens. Spat. Inf. Sci. 2006, $36,5$.

29. Schilling, A.; Schmidt, A.; Maas, H.-G. Automatic Tree Detection and Diameter Estimation in Terrestrial Laser Scanner Point Clouds. In Proceedings of the 16th Computer Vision Winter Workshop, Mitterberg, Austria, 2-4 February 2011; pp. 75-83.

30. Lindberg, E.; Holmgren, J.; Olofsson, K.; Olsson, H. Estimation of stem attributes using a combination of terrestrial and airborne laser scanning. Eur. J. For. Res. 2012, 131, 1917-1931. [CrossRef]

31. Olofsson, K.; Holmgren, J.; Olsson, H. Tree Stem and Height Measurements using Terrestrial Laser Scanning and the RANSAC Algorithm. Remote Sens. 2014, 6, 4323-4344. [CrossRef]

32. Király, G.; Brolly, G. Modelling single trees from terrestrial laser scanning data in a forest reserve. Photogramm. J. Finl. 2008, 21, 37-50.

33. Aschoff, T.; Spiecker, H. Algorithms for the automatic detection of trees in laser scanner data. Int. Arch. Photogramm. Remote Sens. Spat. Inf. Sci. 2004, 36, W2.

34. Pál, I. Measurements of Forest Inventory Parameters on Terrestrial Laser Scanning Data Using Digital Geometry and Topology. Int. Arch. Photogramm. Remote Sens. Spat. Inf. Sci. 2008, 37, 373-380.

35. Belton, D.; Moncrieff, S.; Chapman, J. Processing tree point clouds using Gaussian Mixture Models. In Proceedings of the ISPRS annals of the photogrammetry, remote sensing and spatial information sciences, Antalya, Turkey, 1-13 November 2013.

36. Aiteanu, F.; Klein, R. Hybrid tree reconstruction from inhomogeneous point clouds. Vis. Comput. 2014, 30, 763-771. [CrossRef]

37. Åkerblom, M.; Raumonen, P.; Kaasalainen, M.; Casella, E.; Baghdadi, N.; Thenkabail, P.S. Analysis of Geometric Primitives in Quantitative Structure Models of Tree Stems. Remote Sens. 2015, 7, 4581-4603.

38. Simonse, M.; Aschoff, T.; Spiecker, H.; Thies, M. Automatic determination of forest inventory parameters using terrestrial laser scanning. In Proceedings of the ScandLaser Scientific Workshop on Airborne Laser Scanning of Forests, Umeå, Sweden, 3-4 September 2003; Volume 2003, pp. 252-258.

39. Pfeifer, N.; Winterhalder, D.; Gorte, B.G.H. Three-dimensional reconstruction of stems for assessment of taper, sweep and lean based on laser scanning of standing trees. Scand. J. For. Res. 2004, 19, 571-581.

40. Hackenberg, J.; Morhart, C.; Sheppard, J.; Spiecker, H.; Disney, M. Highly Accurate Tree Models Derived from Terrestrial Laser Scan Data: A Method Description. Forests 2014, 5, 1069-1105. [CrossRef]

41. Wezyk, P.; Kozioł, K.; Glista, M.; Pierzchalski, M. Terrestrial laser scanning versus traditional forest inventory first results from the polish forests. In Proceedings of the ISPRS Workshop on Laser Scanning 2007 and SilviLaser 2007, Espoo, Finland, 12-14 September 2007; Volume 36, pp. 424-429.

42. Yan, D.; Wintz, J.; Mourrain, B.; Wang, W.; Bourdon, F.; Godin, C. Efficient and robust tree model reconstruction from laser scanned data points. In Proceedings of the 11th IEEE International conference on Computer-Aided Design and Computer Graphics 2009, Huangshan, China, 19-21 August 2009; pp. 572-576.

43. Moskal, L.M.; Zheng, G. Retrieving forest inventory variables with terrestrial laser scanning (TLS) in urban heterogeneous forest. Remote Sens. 2012, 4, 1-20. [CrossRef]

44. Pfeifer, N.; Winterhalder, D. Modelling of tree cross sections from terrestrial laser scanning data with free-form curves. Int. Arch. Photogramm. Remote Sens. Spat. Inf. Sci. 2004, 36, W2.

45. Liang, X.; Litkey, P.; Hyyppa, J.; Kaartinen, H.; Vastaranta, M.; Holopainen, M. Automatic Stem Mapping Using Single-Scan Terrestrial Laser Scanning. IEEE Trans. Geosci. Remote Sens. 2012, 50, 661-670. [CrossRef]

46. Kankare, V.; Liang, X.; Yu, X.; Hyyppa, J.; Holopainen, M. Automated Stem Curve Measurement Using Terrestrial Laser Scanning. IEEE Trans. Geosci. Remote Sens. 2014, 52, 1739-1748.

47. Kelbe, D.; Romanczyk, P.; Van Aardt, J.; Cawse-Nicholson, K. Reconstruction of 3D tree stem models from low-cost terrestrial laser scanner data. In Laser Radar Technology and Applications XVIII; International Society for Optics and Photonics: Bellingham, WA, USA, 2013; Volume 8731, p. 873106. 
48. McDaniel, M.W.; Nishihata, T.; Brooks, C.A.; Salesses, P.; Iagnemma, K. Terrain classification and identification of tree stems using ground-based LiDAR. J. Field Robot. 2012, 29, 891-910. [CrossRef]

49. Kelbe, D.; Romanczyk, P. Automatic extraction of tree stem models from single terrestrial lidar scans in structurally heterogeneous forest environments. In Proceedings of the 12th International Conference on LiDAR Applications for Assessing Forest Ecosystems 2012, Vancouver, BC, Canada, 16-19 September 2012; pp. 1-8.

50. Hildebrandt, R.; Iost, A. From points to numbers: A database-driven approach to convert terrestrial LiDAR point clouds to tree volumes. Eur. J. For. Res. 2012, 131, 1857-1867. [CrossRef]

51. Aschoff, T.; Thies, M.; Spiecker, H. Describing forest stands using terrestrial laser-scanning. Int. Arch. Photogramm. Remote Sens. Spat. Inf. Sci. 2004, 35, 237-241.

52. Huang, H.; Li, Z.; Gong, P.; Cheng, X.; Clinton, N.; Cao, C.; Ni, W.; Wang, L. Automated Methods for Measuring DBH and Tree Heights with a Commercial Scanning Lidar. Photogramm. Eng. Remote Sens. 2011, 77, 219-227. [CrossRef]

53. Xi, Z.; Hopkinson, C.; Chasmer, L. Automating Plot-Level Stem Analysis from Terrestrial Laser Scanning. Forests 2016, 7, 252. [CrossRef]

54. Ravaglia, J.; Bac, A.; Fournier, R.A. Extraction of tubular shapes from dense point clouds and application to tree reconstruction from laser scanned data. Comput. Graph. 2017, 66, 23-33. [CrossRef]

55. Côté, J.-F.; Fournier, R.A.; Luther, J.E. Validation of L-Architect model for balsam fir and black spruce trees with structural measurements. Can. J. Remote Sens. 2013, 39, S41-S59. [CrossRef]

56. Raumonen, P.; Kaasalainen, M.; Akerblom, M.; Kaasalainen, S.; Kaartinen, H.; Vastaranta, M.; Holopainen, M.; Disney, M.; Lewis, P. Fast Automatic Precision Tree Models from Terrestrial Laser Scanner Data. Remote Sens. 2013, 5, 491-520. [CrossRef]

57. Raumonen, P.; Casella, E.; Calders, K.; Murphy, S.; Åkerbloma, M.; Kaasalainen, M. Massive-Scale Tree Modelling from TLS Data. ISPRS Ann. Photogramm. Remote Sens. Spat. Inf. Sci. 2015, II-3/W4, 189-196. [CrossRef]

58. Hackenberg, J.; Wassenberg, M.; Spiecker, H.; Sun, D. Non Destructive Method for Biomass Prediction Combining TLS Derived Tree Volume and Wood Density. Forests 2015, 6, 1274-1300. [CrossRef]

59. Hackenberg, J.; Spiecker, H.; Calders, K.; Disney, M.; Raumonen, P. SimpleTree-An Efficient Open Source Tool to Build Tree Models from TLS Clouds. Forests 2015, 6, 4245-4294. [CrossRef]

60. Amanatides, J.; Woo, A. A Fast Voxel Traversal Algorithm for Ray Tracing; Eurographics: Montreal, QC, Canada, 1987; Volume 87, pp. 3-10.

61. Kass, M.; Witkin, A.; Terzopoulos, D. Snakes: Active contour models. Int. J. Comput. Vis. 1988, 1, $321-331$. [CrossRef]

62. Ravaglia, J.; Bac, A.; Fournier, R.A. Anisotropic Octrees: A Tool for Fast Normals Estimation on Unorganized Point Clouds. In Proceedings of the Winter School of Computer Graphics (WSCG) 2017, Plzen, Czech Republic, 29 May-2 June 2017.

63. Pfeifer, N.; Gorte, B.; Winterhalder, D. Automatic Reconstruction of Single Trees From Terrestrial Laser Scanner Data. In Proceedings of the 20th ISPRS Congress, Istanbul, Turkey, 12-23 July 2004; pp. 114-119.

64. Pôle Recherche Développement Innovation, Office National des Forêts. Computree Official Website. 2019. Available online: http://computree.onf.fr/?lang=en (accessed on 5 August 2017).

65. Pharr, M.; Jakob, W.; Humphreys, G. Physically Based Rendering: From Theory to Implementation; Morgan Kaufmann: Burlington, MA, USA, 2016.

66. Saucier, J.-P.; Grondin, P.; Robitaille, A.; Bergeron, J.-F. Zone de Végétation des Domaines Bioclimatiques du Québec; Ministère des Ressources Naturelles, Direction des Inventaires Forestiers: Quebec, QC, Canada, 2001.

67. Pitkänen, T.P.; Raumonen, P.; Kangas, A. Measuring stem diameters with TLS in boreal forests by complementary fitting procedure. ISPRS J. Photogramm. Remote Sens. 2019, 147, 294-306. [CrossRef]

68. Calders, K.; Newnham, G.; Burt, A.; Murphy, S.; Raumonen, P.; Herold, M.; Culvenor, D.; Avitabile, V.; Disney, M.; Armston, J.; et al. Nondestructive estimates of above-ground biomass using terrestrial laser scanning. Methods Ecol. Evol. 2015, 6, 198-208. [CrossRef]

69. Wang, P.; Li, R.; Bu, G.; Zhao, R. Automated low-cost terrestrial laser scanner for measuring diameters at breast height and heights of plantation trees. PLOS ONE 2019, 14, e0209888. [CrossRef]

70. Zhang, W.; Wan, P.; Wang, T.; Cai, S.; Chen, Y.; Jin, X.; Yan, G. A Novel Approach for the Detection of Standing Tree Stems from Plot-Level Terrestrial Laser Scanning Data. Remote Sens. 2019, 11, 211. [CrossRef] 
71. Kunz, M.; Hess, C.; Raumonen, P.; Bienert, A.; Hackenberg, J.; Maas, H.-G.; Härdtle, W.; Fichtner, A.; Oheimb, G. Comparison of wood volume estimates of young trees from terrestrial laser scan data. iForest Biogeosci. For. 2017, 10, 451-458. [CrossRef]

72. Gonzalez de Tanago, J.; Lau, A.; Bartholomeus, H.; Herold, M.; Avitabile, V.; Raumonen, P.; Martius, C.; Goodman, R.C.; Disney, M.; Manuri, S.; et al. Estimation of above-ground biomass of large tropical trees with terrestrial LiDAR. Methods Ecol. Evol. 2018, 9, 223-234. [CrossRef]

73. Maas, H.-G.; Bienert, A.; Scheller, S.; Keane, E. Automatic forest inventory parameter determination from terrestrial laser scanner data. Int. J. Remote Sens. 2008, 29, 1579-1593. [CrossRef]

74. Leblanc, S.; Fournier, R. Hemispherical photography simulations with an architectural model to assess retrieval of leaf area index. Agric. For. Meteorol. 2014, 194, 64-76. [CrossRef]

75. Côté, J.-F.; Fournier, R.A.; Luther, J.E.; Van Lier, O.R. Fine-scale three-dimensional modeling of boreal forest plots to improve forest characterization with remote sensing. Remote Sens. Environ. 2018, 219, 99-114. [CrossRef]

76. Rusu, R.B.; Cousins, S. 3D is here: Point Cloud Library (PCL). In Proceedings of the IEEE International Conference on Robotics and Automation, Shanghai, China, 9-13 May 2011.

77. Boulch, A.; Marlet, R. Deep Learning for Robust Normal Estimation in Unstructured Point Clouds. Comput. Graph. Forum 2016, 35, 281-290. [CrossRef]

78. Brodu, N.; Lague, D. 3D terrestrial lidar data classification of complex natural scenes using a multi-scale dimensionality criterion: Applications in geomorphology. ISPRS J. Photogramm. Remote Sens. 2012, 68, 121-134. [CrossRef]

79. Martin-Ducup, O.; Schneider, R.; Fournier, R.A. Analyzing the Vertical Distribution of Crown Material in Mixed Stand Composed of Two Temperate Tree Species. Forests 2018, 9, 673. [CrossRef]

(C) 2019 by the authors. Licensee MDPI, Basel, Switzerland. This article is an open access article distributed under the terms and conditions of the Creative Commons Attribution (CC BY) license (http://creativecommons.org/licenses/by/4.0/). 